Journal of the Mathematical Society of Japan Vol. 3, No. 1, May, 1951.

\title{
A Note on Finite Ring Extensions
}

\author{
Emil Artin and John T. TAte
}

Let $R \subset S$ be two commutative rings. We shall say that $S$ is a modul finite extension of $R$ if a finite number of elements $\omega_{1}, \omega_{2}, \cdots \omega_{n}$ of $S$ can be found such that

$$
S=R \omega_{1}+R \omega_{2}+\cdots+R \omega_{n}
$$

This modul finite extension has to be distinguished from what we shall call a ring finite extension

$$
S=R\left[\xi_{1}, \xi_{2}, \cdots \xi_{n}\right],
$$

in which every element of $S$ can be written as polynomial in the generators $\xi_{1}, \xi_{2}, \cdots \xi_{n}$ with coefficients in $R$. If we call $S^{\prime}$ the ring of all polynomials in the indeterminates $x_{1}, x_{2}, \cdots x_{n}$ with coefficients in $R$ then $S$ is a homomorphic image of $S^{\prime}$ and the following well known lemma is immediate :

Lemma 1. If $R$ is a Noetherian ring ${ }^{1)}$ with unit element and $S=R\left[\xi_{1}, \xi_{2}, \cdots \xi_{n}\right]$ a ring finite extension of $R$ then $S$ is Noetherian.

Lımma 2. Let $R$ be a Noetherian ring with unit element and $S=R \omega_{1}$ $+R\left(\omega_{2}+\cdots+R\left(\omega_{n}\right.\right.$ a modul finite extension of $R$. Then any intermediate ring $T: R \subset T \subset S$ is also a modul finite extension of $R$.

The proof is simple and also well known. We consider $S$ as an $R$ space. The $R$-subspaces of $S$-and $T$ is one of them-satisfy the ascending chain condition. $T$ is therefore a modul finite extension of $R$.

The main result of our note is: .

Theorcm 1. Let $R$ be a Noetherian ring with unit element, $S=R\left[\xi_{1}\right.$, $\left.\xi_{2}, \cdots \xi_{n}\right]$ a 1 ing finite extension and $T$ an intermediate ring such that. $S$ is a modul finite extension of $T: S=T \omega_{1}+T \omega_{2}+\cdots+T \omega_{m}$. Then $T$ is a ring finite extension of $R$.

Proof: There exist expressions of the form:

$$
\xi_{i}=\sum_{\nu=1}^{m} a_{i \nu} \omega_{\nu} ; \quad i=1,2, \cdots n ; \quad a_{i \nu} \in T
$$

1) i.e. a ring with ascending chain condition for ideals. 


$$
\omega_{i} \omega_{j}=\sum_{\nu=1}^{m} b_{i j \nu} \omega_{\nu} ; i, j=1,2, \cdots m ; \quad b_{i j \nu} \in T .
$$

Let $T_{0}$ be the ring finite extension of $R$ generated by the $a_{i \nu}$ and the $h_{i j \nu}$ Lemma 1 shows that $T_{0}$ is Noetherian. Tivially $T_{0} \subset T \subset S$.

An element of $S$ is a polynomial in the $\xi_{i}$ with coefficients in $R$. Substituting (1) and making repeated use of (2) shows that

$$
S=T_{0} \omega_{1}+T_{0} \omega_{2}+\cdots+T_{0} \omega_{m},
$$

so that $S$ is a modul finite extension of $T_{0}$. Because of lemma 2 our ring $T$ is also a modul finite extension of $T_{0}$, say by e ements $\alpha_{1}, \alpha_{2}, \cdots \alpha_{p}$ of $T$. Therefore $T$ is a ring firite extension of $R$ by the elements $a_{i v}$, $b_{i j \nu}$ and $\alpha_{\nu}$.

As an application we prove the following theorem of Zariski..)

Theorem 2. Let $k$ be a field and assume that the ring finite extension $E=k\left[\xi_{1}, \xi_{2}, \cdots \xi_{n}\right]$ is a field. Then $E / k$ is algebraic and consequently modul finite.

Proof: Suppose $E / k$ is transcendental. Let $\xi_{1}, \xi_{2}, \ldots \xi_{r}$ be algebraically independent, all other $\xi_{v}$ algebraically dependent on $\xi_{1}, \xi_{2}, \ldots \xi_{r}$. Call $F$ the field $k\left(\xi_{1}, \xi_{2}, \cdots \xi_{r}\right)$ of all rational functions of $\xi_{1}, \xi_{2}, \ldots \xi_{r}$. Then $k \subset F \subset E$ and $E$ is a modul finite extension of $F$ (being a finite algebraic extension of $F$ ). Because of theorem $1 F$ would be a ring finite extension $k\left[\eta_{1}, \eta_{2}, \cdots \eta_{m}\right]$ of $k$. Each $\eta_{i}$ is a rational function of $\xi_{1}, \xi_{2}, \ldots \xi_{r}$. Let $M$ be the set of all denominators of the $\eta_{i}$. In the polynomial domain $k\left[\xi_{1}\right.$, $\left.\boldsymbol{\xi}_{2}, \cdots \boldsymbol{\xi}_{\boldsymbol{r}}\right]$ there are infinitely many irreducible polynomials. (One can make a uniform proof for all fields $k$ which is similar to Euclid's proof for the existence of infinitely many primes.) Let $f$ be irreducible and assume $f$ divides none of the polynomials of $M$. The element $\frac{1}{f}$ of $F$ could not be a polynomial in $\eta_{1}, \eta_{2}, \cdots \eta_{m}$. This is a contradiction.

Zariski uses theorem 2 for a short proof of Hilbert's Nullstellensatz. He concludes as follows :

Let $\mathfrak{a} \neq \mathfrak{p}$ be an ideal in the domain of polynomials $\mathfrak{D}=k\left[x_{1}, x_{2}, \cdots x_{n}\right]$ in indeterminates $x_{\nu}$. Let $\mathfrak{p} \supset \mathfrak{a}$ be a maximal ideal above $\mathfrak{a}$. Then $\mathfrak{o}_{/} \mathfrak{p}$ is a field on oxe hand and a ring finite extension of $k$ by the residue

2) Oscar Zariski, A new proof of Hilbert's Nullstellensatz. Bull. Amer. Math. Soc. 53 (1947). 
classes $\mu_{1}, \mu_{2}, \cdots \mu_{n}$ of $x_{1}, x_{2}, \cdots x_{n}$ on the other. Therefore each $\mu_{i}$ is algebraic over $k$. If $f\left(x_{1}, x_{2}, \cdots x_{i}\right) \in \mathfrak{p}$ then $f\left(\mu_{1}, \mu_{2}, \cdots \mu_{n}\right)=0$. Therefore $\mathfrak{p}$ has an algebraic zero and a fortiori $\mathfrak{a}$.

If consequently $\mathfrak{a}$ is an ideal without algebraic zeros then $a=\mathfrak{b}$. The full Nullstellensatz is an easy consequence of this statement. ${ }^{3)}$

Now let $R$ be a Noetherian integral domain with unit element 1 and quotient field $F$.

Theorem 3. $R$ has a ring finite extension $S=R\left[\xi_{1}, \xi_{2}, \cdots, \xi_{n}\right]$ which is a field, if and only if $F$ is itself a ring finite extension of $R$. If this is the case the fields of type $S$ are simply all modul finite extension fields of $F$.

Proof: If $S$ is a field, then $R \subset F \subset S$ and $S=F\left[\xi_{1}, \xi_{2}, \ldots \xi_{n}\right]$. According to theorem $2 S$ is a modul finite extension of $F$. From theorem 1 it follows that $F$ is $\mathrm{s}$ ring finite extension of $R$. Conversely, if $F$ is a ring finite extension, then any modul finite extension of $F$ is obviously a ring finite extension of $R$.

Our next theorcm gives necessary and sufficient conditions for $F$ to be a ring finite extension of $R$.

Theorcm 4. The following four statements about $R$ are equivalent:

(A) $F$ is a ring finite extension of $R$.

- (B) There exists an element $a \neq 0$ of $R$ which is contained in all prime ideals of $R$.

(C) There are only a finite number of minimal prime ideals of $R$.

(D) There are only a finite number of prime ideals in $R$, and every one of them is maximal.

(By ideal we always mean a "proper" ideal, different from $\{0\}$ and $R$.) Proof :

$(\mathrm{A}) \rightarrow(\mathrm{B}):$ Let $F=R\left[\eta_{1}, \eta_{2}, \cdots, \eta_{n}\right]$. Let $a \in R$ be a common denominator of the $\eta_{i}$. Then for any element $f=f\left(\eta_{1}, \eta_{2}, \cdots, \eta_{i n}\right) \in F$ we have $a^{\nu} f \in R$ for some $\nu$. Given any prime ideal $\mathfrak{p}$ of $R$, let $b \neq 0$ be an element of $\mathfrak{p}$. Then we have $a^{\nu} \frac{1}{b} \in R$; hence $a^{\nu} \in b R \subset \mathfrak{p}$ and therefore $a \in \mathfrak{p}$.

$(B) \rightarrow(C)$ : Let $a R=q_{1} \cap q_{2} \cap \cdots \cap \mathfrak{q}_{r}$, each $\mathfrak{q}_{i}$ primary belonging to $\mathfrak{p}_{i}$. For a sufficiently high $m$ we have $\mathfrak{p}_{i}^{m} \subset q_{i}$ for all $i$. Let $\mathfrak{p}$ be any prime icleal. Then

3) See for instance : van der Waerden, Moderne Algebra, vol. 2 (1931), p. 11. 


$$
\mathfrak{p}_{1}^{m} \mathfrak{p}_{2}^{m} \cdots \mathfrak{p}_{r}^{m} \subset \mathfrak{q}_{1} \mathfrak{q}_{2} \cdots \mathfrak{q}_{r} \subset \mathfrak{q}_{1} \cap \mathfrak{q}_{2} \cap \cdots \cap \mathfrak{q}_{r}=a R \subset \mathfrak{p},
$$

and therefore $\mathfrak{p}_{i} \subset \mathfrak{p}$ for some $i$. It follows that the minimal primes must be among the primes $\mathfrak{p}_{1}, \mathfrak{p}_{2}, \cdots, \mathfrak{p}_{r}$.

$(\mathrm{C}) \rightarrow(\mathrm{D})$ : We shall use the fact that any element $c \in R$ which is not a unit is contained in some minimal prime. This follows directly from a theorem of $\mathrm{Krull}^{4}$ ) which states that any prime ideal which is minimal among the primes containing a principal ideal $c R$ is minimal in $R$.

Let $\mathfrak{p}_{1}, \mathfrak{p}_{2}, \cdots, \mathfrak{p}_{s}$ be the minimal primes of $R$. For each $i$, there exists an element $a_{i} \notin \mathfrak{p}_{i}$ such that $a_{i} \in \mathfrak{p}_{j}$ for $j \neq i$. Otherwise we would have $\mathfrak{p}_{i} \supset \cap_{j \neq i} \mathfrak{p}_{j} \supset \Pi_{j \neq i} \mathfrak{p}_{j}$, and therefore $\mathfrak{p}_{i} \supset \mathfrak{p}_{j}$ for some $j \neq i$, contradicting the minimality of $\mathfrak{p}_{i}$. Take now any element $b \notin \mathfrak{p}_{1}$. The element

$$
b^{\prime}=b+\sum_{i ; b \in \mathfrak{p}_{i}} a_{i} \equiv b\left(\bmod \mathfrak{p}_{1}\right)
$$

is clearly contained in none of the minimal primes $\mathfrak{p}_{i}$ and is therefore a unit. It follows that $\mathfrak{p}_{1}$, and similarly any $\mathfrak{p}_{i}$, is maximal.

$(\mathrm{D}) \rightarrow(\mathrm{C}):$ Trivially.

$(\mathrm{C}) \rightarrow(\mathrm{B}):$ Take an $a \neq 0$ in the product of the minimal primes.

$(B) \rightarrow(A):$ Take $b \neq 0$ in $R$. Write $b R=\mathfrak{q}_{1} \cap \mathfrak{q}_{2} \cap \cdots \cap \mathfrak{q}_{r}$, each $\mathfrak{q}_{i}$ primary belonging to $\mathfrak{p}_{i}$. From $a \in \mathfrak{p}_{i}$ we conclude some power of $a$ is in all the $q_{i}$, therefore in $b R: a^{m}=b c$. Then $\frac{1}{b}=\frac{c}{a^{m}}$ shows that $F=R\left[\frac{1}{a}\right]$.

The question whether a field $E \supset R$ can be imbedded in a ring finite extension $S=R\left[\xi_{1}, \xi_{2}, \cdots, \xi_{n}\right]$ of $R$ can be answered immediately. Let $\mathfrak{p}$ be a maximal ideal of $S$. The residue class field $S / \mathfrak{p}$ still contains $E$ and $S / \mathfrak{p}=R\left[\eta_{1}, \eta_{2}, \cdots, \eta_{n}\right]$ where $\eta_{i}$ is the residue class of $\xi_{i}$. According to theorem $3 R$ has to satisfy the condition stated in this theorem and $S / \mathfrak{p}$ is a modul finite extension of $F$. Therefore $E$ is a modul finite extension of $F$.

\section{Princeton University.}

4) W. Krull, Dimensionstheorie in Stellenringen, Journal für die reine und angewandte Mathematik, vol. 179, p. 221 (1938). 